OPEN ACCESS

Edited by:

Hailiang Tang,

Fudan University, China

Reviewed by:

Bharat Guthikonda,

Louisiana State University Health

Shreveport, United States

Gustavo Isolan,

Centro Avançado de Neurologia e

Neurocirurgia (CEANNE), Brazil

*Correspondence:

Tao Li

taoli_kh@163.com

Shuang Hu

hushuangkh@163.com

Specialty section:

This article was submitted to

Neuro-Oncology and

Neurosurgical Oncology,

a section of the journal

Frontiers in Oncology

Received: 19 August 2021 Accepted: 04 November 2021 Published: 22 November 2021

Citation:

Gao B, Zhang Y, Tan J, Ouyang J,

Tai B, Cao X, LiT and Hu S (2021) Surgical Treatment and Clinical Outcomes of Petroclival Meningiomas: A Single-Center Experience of 107 Patients. Front. Oncol. 11:761284. doi: 10.3389/fonc.2021.761284

\section{Surgical Treatment and Clinical Outcomes of Petroclival Meningiomas: A Single-Center Experience of 107 Patients}

\author{
Baocheng Gao ${ }^{1}$, Yongfa Zhang ${ }^{1}$, Jiang Tan ${ }^{1}$, Jinsong Ouyang ${ }^{1}$, Bai Tai ${ }^{1}$, Xianbao Cao ${ }^{2}$, \\ Tao $\mathrm{Li}^{1 *}$ and Shuang $\mathrm{Hu}^{2 *}$ \\ ${ }^{1}$ Department of Neurosurgery, The First People's Hospital of Yunnan Province, The Affiliated Hospital of Kunming University \\ of Science and Technology, Kunming, China, ${ }^{2}$ Department of Ear, Nose and Throat (ENT) and Head and Neck (HN) Surgery, \\ The First People's Hospital of Yunnan Province, The Affiliated Hospital of Kunming University of Science and Technology, \\ Kunming, China
}

Objective: This study aimed to establish optimal surgical strategies via reviewing the clinical outcomes of various surgical approaches for the pertroclival meningiomas (PCMs).

Methods: This retrospective study enrolled 107 patients with PCMs at the authors' institution from year 2010 to 2020. Patient demographics, the clinical characteristics, various operative approaches, major morbidity, post-operative cranial nerve deficits and tumor progression or recurrence were analyzed.

Results: The subtemporal transtentorial approach (STA), the Kawase approach (KA), the retrosigmoid approach (RSA) and the anterior sigmoid approach (ASA), namely the posterior petrosal approach (PPA) were adopted for 17 cases, 22 cases, 31 cases and 34 cases respectively. Total or subtotal resection was achieved in 96 cases (89.7\%). The incidence of new-onset and aggravated cranial nerve dysfunction were 13.1\% (14/107) and $10.4 \%$ (15/144), respectively. Furthermore, 14 cases suffered from intracranial infection, 9 cases had cerebrospinal fluid leakage, and 3 cases sustained intracranial hematoma ( 1 case underwent second operation). The mean preoperative and postoperative Karnofsky Performance Status (KPS) score was 80 (range 60-100) and 78.6 (range 0-100), but this was not statistically significant $(P>0.05)$. After a mean followup of 5.1 years (range 0.3-10.6 years), tumor progression or recurrence was confirmed in 23 cases. Two cases died from postoperative complications.

Conclusions: For the treatment of PCMs, it is still a challenge to achieve total resection. With elaborate surgical plans and advanced microsurgical skills, most patients with PCMs can be rendered tumor resection with satisfactory extent and functional preservation, despite transient neurological deterioration during early postoperative periods.

\footnotetext{
Keywords: petroclival, meningioma, surgical approach, skull base, neuronal function
} 


\section{INTRODUCTION}

Petroclival meningiomas (PCMs) refer to meningiomas that occur on the upper two-thirds of the clivus and medially to the internal auditory canal (IAC), adjacent to the major neurovascular structures, including brainstem, basilar artery, perforating arteries, and III-VII cranial nerves (CN) (1). Since most PCMs are World Health Organization (WHO) grade I tumors, the treatment goal should be curative total resection (TR) during the first operation when the arachnoid membranes are intact. In terms of the surgical approaches, the subtemporal transtentorial approach (STA) and the Kawase approach (KA) have the characteristics of short operative distance, convenient tumor base resection and less intraoperative bleeding but with the limitation for large posterior petrosal PCMS; the retrosigmoid approach (RSA) has the feature of fewer approach-related complications while the restriction to the petroclival region; the anterior sigmoid approach (ASA), also named the posterior petrosal approach (PPA), can facilitate the exposure of tumors, reduce the traction of the brainstem but perplexes the neurosurgeons for harder maneuver. Although there are many surgical approaches for PCMs and the relevant studies have repeatedly reported, the optimal choice for the operation is of extreme difficulty (2-5) and no uniform standard establishing the superiority of one approach over another is acknowledged currently $(6,7)$, due to the anatomical complexity, the multiformity of tumor invasion and the intricacy of a balance between neurofunctional preservation and tumor recurrence.

Despite remarkable advances in microsurgical techniques during the past decades, many recent reports (1) still reveal a low TR rate and suggest that aggressive extirpation is often associated with severe morbidity. For this reason, some authors proposed subtotal resection (STR) followed by radiotherapy in order to preserve the neurological functions. However, others insist that aggressive resection using various skull base surgical techniques should guarantee more favorable outcomes and the control of highgrade tumors. The surgical treatment of PCMs has always been a challenge for skull base neurosurgeons due to the deep location, complex adjacent structures and their scarcity (less than $0.15 \%$ of all intracranial meningiomas) (1). This study aimed to establish optimal surgical strategies via reviewing the clinical outcomes of various surgical approaches for PCMs based on our 107 cases.

\section{MATERIALS AND METHODS}

\section{Patients}

The present study was approved by the Ethics Committee of the First People's Hospital of Yunnan Province. All procedures performed in studies that involved human participants were in accordance with the ethics standards of the Institutional and National Research Committee, and the 1964 Helsinki Declaration and its later amendments or comparable ethics standards. Written consent was obtained from the patients.

This retrospective study enrolled 107 patients from the Department of Neurosurgery, the First People's Hospital of Yunnan Province, from January 2010 and December 2020. All patients were diagnosed with PCMs based on radiological and histopathological results. The exclusion criteria were as follows: (1) patients with a magnetic resonance imaging (MRI) revealing that the main body of the tumor is located on the inferior clivus or lateral wall of the IAC; (2) patients with multiple $(\geq 2)$ intracranial meningiomas; (3) patients with no successful follow-up.

\section{Clinical and Radiological Evaluation}

The demographic and clinical profiles were collected, and the operative logs were reviewed. The neurological functions were evaluated preoperatively and 2 weeks after operation using the Karnofsky Performance Status (KPS) scale, and all patients underwent perioperative MRI scans. The extent of the resection was determined based on the intraoperative finding and enhanced MRI within 72 hours postoperatively. Total resection was defined as Simpson grade I and II, subtotal resection was defined as Simpson grade III and IV, and partial resection was defined as Simpson grade V. According to the classification system proposed by Kawase et al. (8) in 1996 and Ichimura et al. (9) in 2008, the PCMs were divided into four groups: upper clival type, cavernous type, tentorium type, and petrous apex type. According to the size-based classification criteria proposed by Sekhar et al. (10), measured on the maximum diameter, tumors were small $(<10 \mathrm{~mm})$, medium (10-24 mm), large (25-44 $\mathrm{mm})$, and giant $(\geq 45 \mathrm{~mm})$.

\section{Surgical Treatment}

The surgical resection was performed with the assistance of electrophysiological monitoring, and the selection of surgical approaches was shown in Table 1. For petrous apex type PCMs,

TABLE 1 | Selection of surgical approaches based on imaging classification.

\begin{tabular}{|c|c|c|c|c|c|c|c|c|c|}
\hline \multirow[t]{2}{*}{ Imaging classification } & \multirow[t]{2}{*}{ Cases (n) } & \multicolumn{5}{|c|}{ Approach (n) } & \multirow[t]{2}{*}{$\operatorname{TR}(n)$} & \multirow[t]{2}{*}{ SR $(n)$} & \multirow[t]{2}{*}{$\operatorname{PR}(n)$} \\
\hline & & STA & KA & RSA & ASA & CA & & & \\
\hline PAT & 16 & 11 & 5 & 0 & 0 & 0 & 16 & 0 & 0 \\
\hline$\pi$ & 38 & 6 & 0 & 19 & 13 & 0 & 15 & 23 & 0 \\
\hline CT & 19 & 0 & 13 & 5 & 0 & $1^{a}$ & 0 & 10 & 9 \\
\hline UCT & 34 & 0 & 4 & 7 & 21 & $2^{b}$ & 26 & 6 & 2 \\
\hline SUM & 107 & 17 & 22 & 31 & 34 & 3 & 57 & 39 & 11 \\
\hline
\end{tabular}

PAT, petrous apex type; TT, tentorium type; CT, Cavernous type; UCT, upper clivus type; SUM, summation; STA, subtemporal transtentorium approach; KA, Kawase approach; RSA, retrosigmoid approach; ASA, anterior sigmoid approach; CA, combined posterior and anterior petrosal approach; TR, total resection; SR, subtotal resection; PR, partial resection.

aThe Fisch's type A approach was used, because the tumor invaded the infratemporal fossa.

${ }^{b}$ The combined posterior and anterior petrosal approach was used, because the tumors extensively invaded the cavernous sinus and crossed the midline of the clivus. 
the subtemporal transtentorium approach (STA) was preferred. However, when the tumor was large, the Kawase approach (KA) was used. For cavernous type PCMs, KA was the first choice. However, when the tumor was located in the posterior fossa and barely invaded the middle fossa, the retrosigmoid approach (RSA) was used. For upper clivus type PCMs, the anterior sigmoid sinus approach (ASA) was adopted. However, when the tumor did not cross the midline of the clivus, the KA or RSA were selected. For tentorium type PCMs, the STA was used when the tumor size was small or medium, while the tumor was large or giant the RSA or ASA should be used depending on whether the tumor crossed the midline of the clivus.

\section{Follow-Up and Statistical Analysis}

The follow-up was implemented on an outpatient basis. Clinical and radiological examinations were performed. The SPSS 20.0 software (IBM Corp., Armonk, NY, USA) was used for the statistical analysis. The KPS scores was compared using $t$-test, and the progression or recurrence rate was evaluated using a chisquare test (or a Fisher exact test when necessary). And the probability $(P)$ values $<0.05$ were considered statistically significant.

\section{RESULTS}

\section{Demographic Characteristics}

There were 28 males (26.2\%) and 79 females (73.8\%), with an average age of 42.8 years old (range 19-72 years). The clinical symptoms were as follows: headache in 33 cases (30.8\%); CN III, IV, and/or VI dysfunction (diplopia) in 25 cases (23.4\%); CN V dysfunction (trigeminal neuralgia and/or facial numbness) in 61 cases (57.0\%); CN VIII dysfunction (hearing impairment) in 28 cases $(26.2 \%)$, CN VII dysfunction (facial paralysis) in 11 cases (10.3\%); posterior group cranial nerves dysfunction in 19 cases (17.8\%); ataxia in 24 cases $(22.4 \%)$, and progressive hemiparesis in 10 cases $(9.3 \%)$. In addition, 13 patients (12.1\%) were asymptomatic, who requested surgical operation due to psychological stress and other factors. The median duration between onset and surgical treatment were 29 months (range 4-156 months). The mean tumor size was $39.1 \mathrm{~mm}$ (range 6.2$75.9 \mathrm{~mm}$ ), in which 59 patients $(55.1 \%)$ had large tumors, 40 cases $(37.4 \%)$ with giant tumors, 6 cases $(5.6 \%)$ had medium tumors, and only 2 patients $(1.9 \%)$ had small tumors. The mean follow-up period was 5.1 years (range $0.3-10.6$ years). The patient characteristics and detailed clinical information are shown in Table 2.

\section{Surgical Approaches and the Extent of Surgical Resection}

STA was used in 17 cases, KA was selected in 22 cases, ASA was adopted in 34 cases, and RSA was employed in 31 cases. The combined posterior and anterior petrosal approach was used in two cases and the Fisch's type A approach was used in one case. A total of 57 patients underwent Simpson grade I or II resection (total resection, Figure 1). Total or subtotal resection was achieved in 96 patients $(89.7 \%)$, whereas subtotal and partial
TABLE 2 | Demographic data for 107 patients with petroclival meningiomas.

\begin{tabular}{lc}
\hline Demographic data & Value \\
\hline Mean age (range, yrs) & $42.8(19-72)$ \\
Male/female ratio & $28: 79$ \\
Mean preoperative KPS score (range) & $80(60-100)$ \\
Mean clinical follow-up (range, yrs) & $5.1(0.3-10.6)$ \\
Symptoms \& signs at onset (no., \%) & \\
Headache & $33(30.8 \%)$ \\
Diplopia & $25(23.4 \%)$ \\
Trigeminal neuralgia and/or facial numbness & $61(57.0 \%)$ \\
Acoustic-facial bundle dysfunction & $39(36.4 \%)$ \\
Posterior cranial nerve dysfunction & $19(17.8 \%)$ \\
Ataxia & $24(22.4 \%)$ \\
Progressive hemiparesis & $10(9.3 \%)$ \\
Asymptomatic and others & $13(12.1 \%)$ \\
Mean tumor size (range, mm) & $39.1(6.2-75.9)$ \\
Small (<10 mm) & $2(1.9 \%)$ \\
Medium (10mm $\leq$ diameter<25mm) & $6(5.6 \%)$ \\
Large (25mm $\leq$ diameter<45mm) & $59(55.1 \%)$ \\
Giant $(\geq 45 m m)$ & $40(37.4 \%)$ \\
\hline
\end{tabular}

resection were achieved in 39 patients and 11 patients respectively. Details of the surgical approaches and the extent of removal were shown in Table $\mathbf{1 .}$

\section{Postoperative Histopathology of the Tumors}

Pathology was reported as WHO grade I in 95 cases (88.8\%), 9 tumors were reported as WHO grade II (atypical type) and 3 as WHO grade III (anaplastic type). Among the 95 cases of grade I meningiomas, the meningothelial subtype (66 cases, 69.5\%) was most common, followed by transitional subtype (17 patients, $17.9 \%$ ), secretory subtypes (6 cases, 6.3\%) and other subtypes (6 cases, $6.3 \%$ ). There was no statistical significance between the $\mathrm{WHO}$ grade and degree of tumor resection ( $\mathrm{P}>0.05$, Figure 2).

\section{Postoperative Complications and Functional Outcome}

The incidence of new-onset and aggravated cranial nerve dysfunction were $13.1 \%(14 / 107)$ and $10.4 \%$ (15/144), respectively. The CN IV to VIII dysfunction was common, and most of them had improved at recent follow-up. Detailed cranial nerve dysfunctions were shown in Table 3. Other major postoperative morbidities were intracranial infection (14 cases, $13.1 \%)$, cerebrospinal fluid leakage (9 patients, $8.4 \%$ ), postoperative hematoma ( 3 patients, $2.8 \%$ ), in which 1 case needed second operation (Figure 3 ). Two patients with postoperative hematoma died of pneumonia and multiple organ failure, respectively. The mean preoperative and postoperative KPS scores were 80 (range 60-100) and 78.6 (range 0-100), respectively. This was not statistically significant $(t=-0.102$, $P=0.922)$. Furthermore, at their most recent follow-up, 57 cases (53.3\%) were stable with no worsened KPS, and 36 cases (33.6\%) had improved, only 14 patients (13.1\%) had aggravated KPS score.

\section{Tumor Progression or Recurrence}

During the follow-up, overall progression or recurrence was confirmed in 23 cases $(21.5 \%)$. According to the extent of 

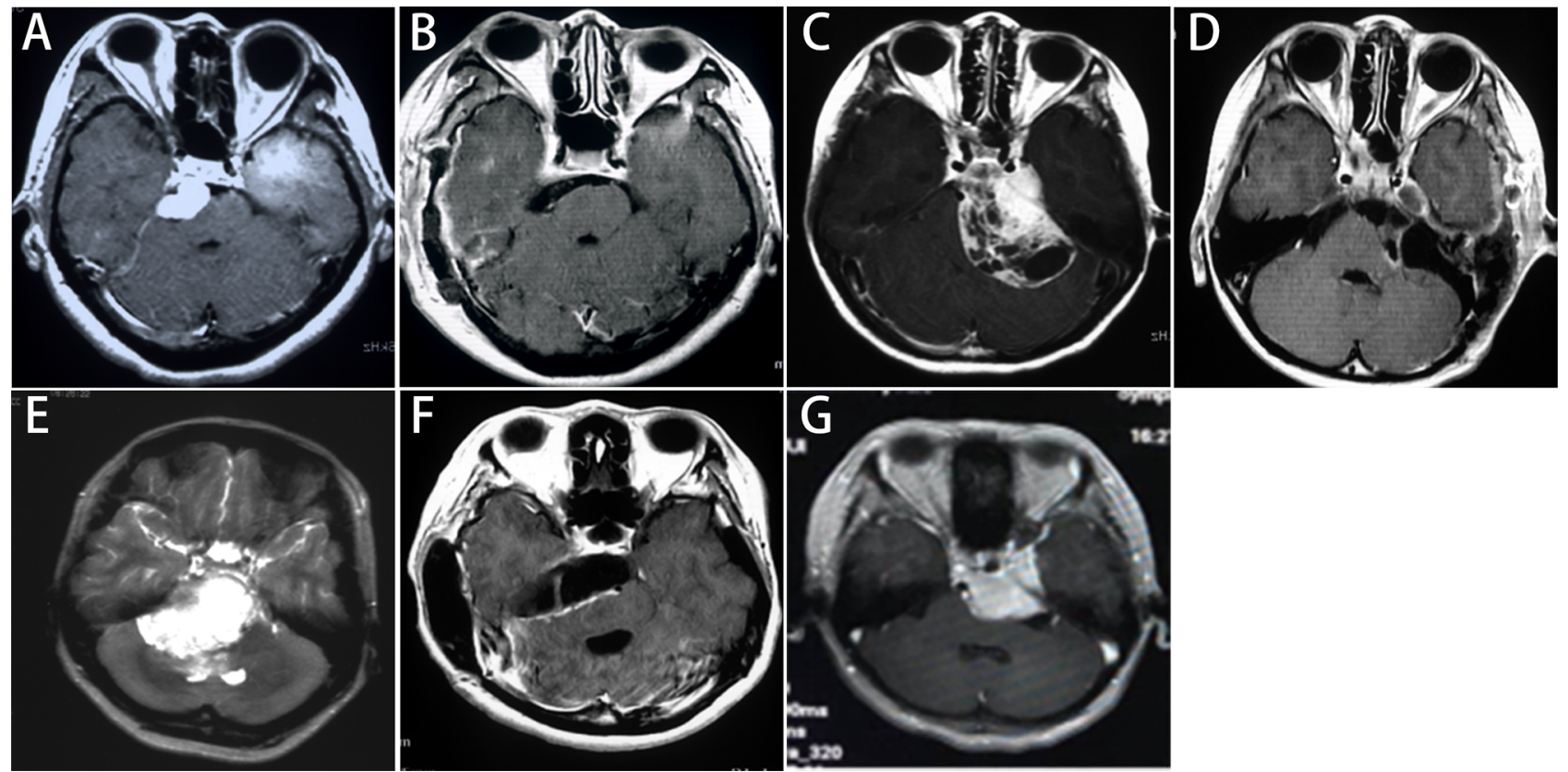

FIGURE 1 | Imaging classification and selection of surgical approaches for PCMs. Petrous apex type: (A) The preoperative enhanced MRI shows that the subtemporal transtentorial approach was used; (B) The MRI within postoperative 72 hours shows a Simpson grade I resection. Tentorium type: (C) The preoperative enhanced MRI shows that the retrosigmoid approach was used; (D) The MRI within postoperative 72 hours shows that the tumor invading the posterior wall of the cavernous sinus was removed, yielding a Simpson grade II resection. Upper clivus type: (E) The preoperative enhanced MRI shows that the anterior sigmoid approach was used; (F) The MRI within postoperative 72 hours shows a Simpson grade II resection. Cavernous type: (G) The preoperative enhanced MRI shows that the Kawase approach was used, and a Simpson grade III resection was achieved.

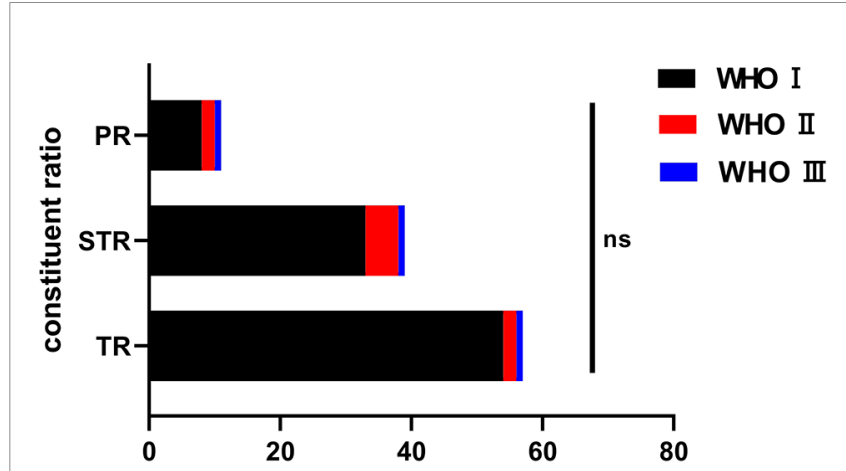

FIGURE 2 | The pathological classification among the groups and the resection degree. ns, no significance.

tumor resection, the progression or recurrence rate was $72.7 \%$ (8 of 11 patients) in the partial resection group, $28.2 \%$ (11 of 39 cases) in the subtotal resection group, and 7\% (4 of 57 patients) in the total resection group. This was statistically significant among groups $(\mathrm{P}<0.05$, Figure 4). According to the pathological subtypes, the progression or recurrence rate was $15.8 \%(15 / 95$ cases), $55.6 \%$ (5/9 patients) and $100 \%$ in the WHO grade I, II and III group, respectively. This was statistically significant when WHO grade I compared with WHO grade II and III $(\mathrm{P}<0.05$, Figure 5).

\section{DISCUSSION}

PCMs usually adjoin the brainstem and cranial nerves. Although the surgical techniques have been greatly improved in recent years, the total resection rate of PCMs remains low (approximately 30-70\%), and the incidence of postoperative complications is approximately $20-30 \%$ (11). Hence surgical management is still one of the most challenging problems for skull base neurosurgeons (12-15), and the selection of surgical approaches for PCMs has become a hot topic $(3,16,17)$. According to the experience of the investigators, petrous apex type PCMs are often located above the trigeminal nerve, which is often displaced by the tumor. Therefore, STA gives priority to surgeons to cut off the tumor base and effectively reduces bleeding. In the study, a total of 17 patients (11 cases of petrous apex type and 6 cases of tentorial type) underwent surgical resection via the STA. It was found that this approach could facilitate the exposure and complete removal of small-tomedium petrous apex type and tentorial type PCMs. Cavernous type PCMs often invade the middle and posterior fossa in a dumbbell-like fashion. The KA can help anteriorly reach the anterior clinoid process (18) and posteriorly reach the plane of the IAC, completely expose the lateral wall of the cavernous sinus, which facilitates the gross total resection. In the present study, a total of 22 cases (13 cases of cavernous type, 5 cases of petrous apex type, and 4 cases of upper clivus type) were treated 
TABLE 3 | Dysfunctions of the cranial nerves.

\begin{tabular}{|c|c|c|c|c|c|c|}
\hline \multirow[t]{2}{*}{ Cranial nerve } & \multirow[t]{2}{*}{ Preoperation } & \multicolumn{4}{|c|}{2 weeks after operation } & \multirow[t]{2}{*}{ Follow-up } \\
\hline & & Unchanged & Aggravated & New-onset & Improved & \\
\hline III & 9 & 4 & 1 & 1 & 4 & 5 \\
\hline IV & 4 & 1 & 2 & 3 & 1 & 3 \\
\hline V & 61 & 17 & 2 & 2 & 42 & 11 \\
\hline $\mathrm{Vl}$ & 12 & 6 & 1 & 1 & 5 & 5 \\
\hline VII & 11 & 3 & 5 & 4 & 3 & 6 \\
\hline VIII & 28 & 13 & 3 & 2 & 12 & 15 \\
\hline$|X-X| \mid$ & 19 & 7 & 1 & 1 & 11 & 2 \\
\hline Sum & 144 & 51 & 15 & 14 & 78 & 47 \\
\hline
\end{tabular}

Sum, summation.
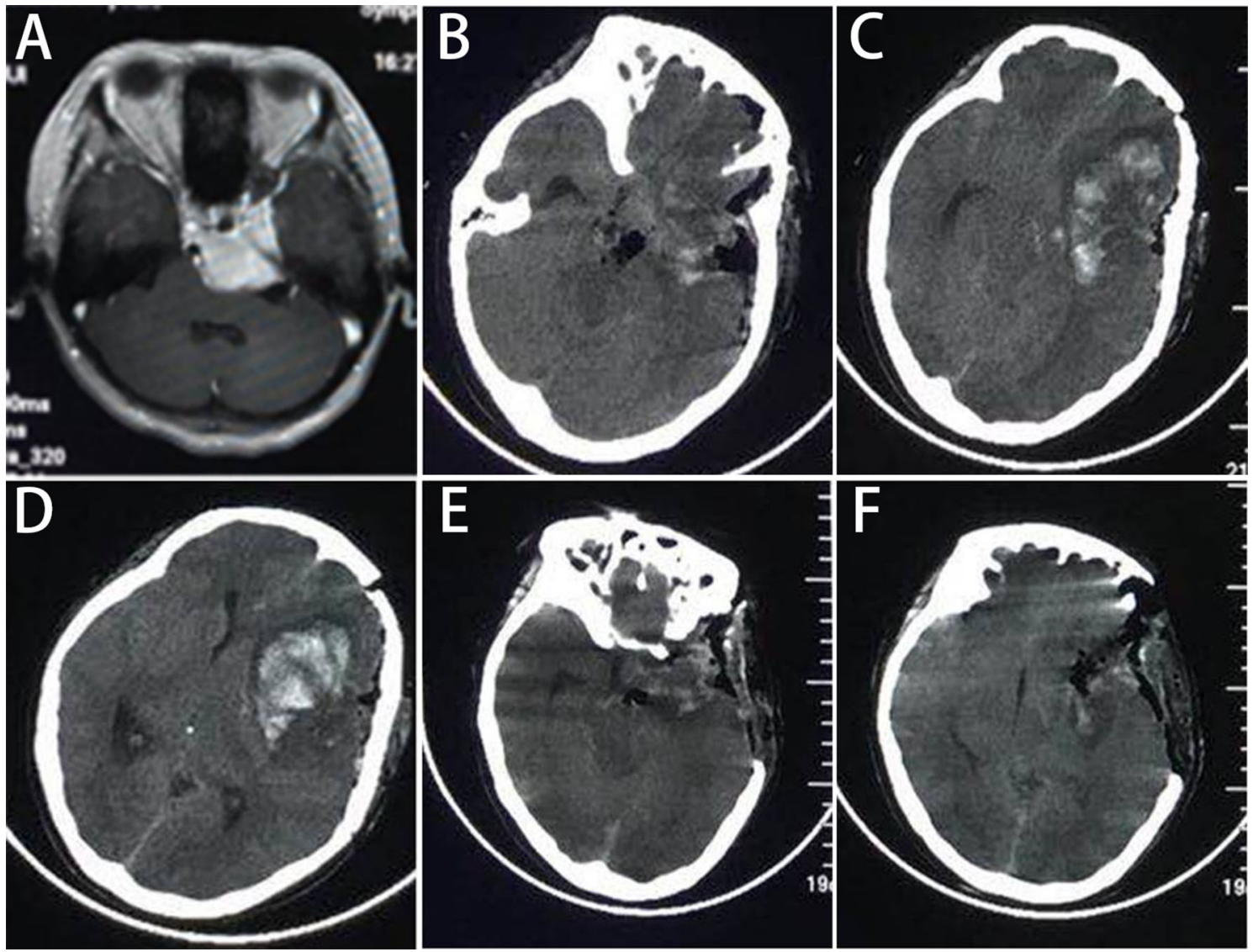

FIGURE 3 | The one patient who had postoperative hematoma and needed second operation. (A) The MR scan shows the cavernous type PCMs, and (B-D) the CT at 6 postoperatively show a hematoma in the frontal lobe with midline displacement. (E, F) The postoperative CT shows that the hematoma was evacuated.

with the KA. The investigators consider the KA to be suitable for the majority of PCMs, especially the cavernous type tumors that straddle the middle and posterior fossa. Upper clivus type PCMs often invade across the midline of the clivus, and the ASA can reduce the traction of the brainstem (19). In this study, a total of 34 patients (21 patients with upper clivus type PCM and 13 patients with tentorium type PCM) were treated with the ASA. The ASA is preferred for large tumors, especially those involving the lateral part of the IAC and midline of the clivus (20).
For tentorium type PCMs, the tumor base is located at the tentorium, and the tumor often grows into the posterior cranial fossa. When the tumor was large to giant, surgical exposure via the STA is limited, whereas the RSA can serve the tumor exposure. In addition, through the RSA, the separation of the tumor from the trigeminal nerve and acoustic and facial nerve was under direct vision, which can effectively reduce the incidence of postoperative complications. Moreover, the tentorium can be incised and the tumor that invades the posterior wall of the cavernous sinus can 


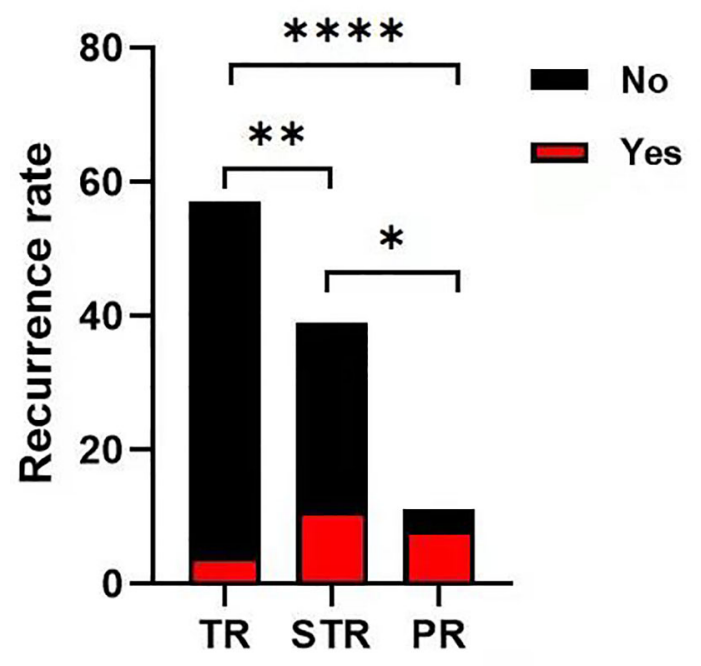

FIGURE 4 | The progression or recurrence rate among the groups. The progression or recurrence rate was $72.7 \%$ (8 of 11 cases) in PR group, $28.2 \%$ (11 of 39 cases) in STR group, 7\% (4 of 57 cases) in TR group, respectively. The differences were statistically significant between each two groups (Chi-square test, $\left.{ }^{\star} p<0.05{ }^{\star \star} p<0.01,{ }^{\star \star \star *} p<0.0001\right)$.

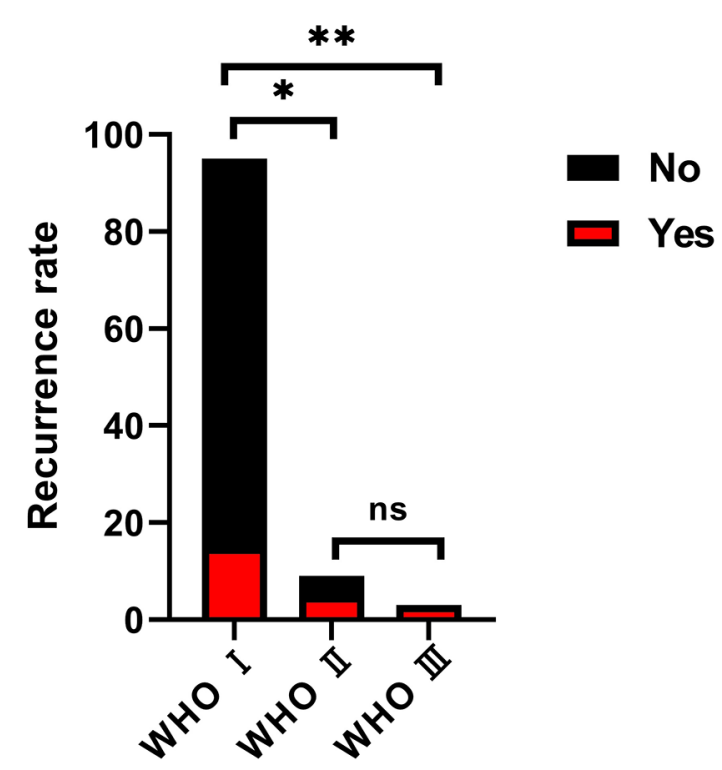

FIGURE 5 | The progression or recurrence rate among groups on the basis of the pathological classification. the progression or recurrence rate was 15.8\% (15/95 cases), 55.6\% (5/9 patients) and 100\% in the WHO grade I, II and III group, respectively. This was statistically significant when $\mathrm{WHO}$ grade I compared with WHO grade II (Chi-square test, ${ }^{*} \mathrm{p}<0.05$ ) and III (Chi-square test, $\left.{ }^{* \star} \mathrm{p}<0.05\right)$, but no significance between grade II and grade III. (Chi-square test, ns, no significance).

also be well-exposed. A total of 31 cases (19 cases of tentorium type, 7 cases of upper clivus type, and 5 cases of cavernous type) were treated using the RSA.
In addition, the selection of a specific surgical approach should be based on the location of the tumor base, tumor size, degree of invasion, and surgeon's familiarity with the approach. If the tumor extensively involved the cavernous sinus and midline of the clivus, the combined posterior and anterior petrosal approach should be applied. In our study, two patients were treated with this combined approach. Additionally, one patient underwent the Fisch A-type approach due to the tumor invasion into the infratemporal fossa. Moreover, preoperative assessment of the venous anatomy for surgical planning is also essential $(21,22)$. We recommend that all patients with PCMs should have MRV or CTV imaging, or/and DSA when it is necessary, before surgery. For example, if the preoperative results show that the Labbé vein flows into the superior petrosal sinus or the patient have a high jugular bulb, the lateral skull base approach (such as ASA) should be avoided prudently. If the tumor invades the middle and posterior fossa, the modified ASA approach is used to protect the superior petrosal sinus $(23,24)$. Likewise, if the Labbe vein drains into the transverse sinus at the anterior part of the temporal lobe, the STA or KA approach is restricted. If the tumor is small and slightly invades the cavernous sinus, we can also use STA or KA. In order to avoid damage to the Labbé vein, we often use mannitol or implant the lumbar cistern drainage to lower intracranial pressure. In the meantime, according to the situation of the Labbe vein during the operation, sharp separation or the removal of part of temporal lobe can be adopted to increase its mobility.

In this study, total or subtotal resection was achieved in 96 cases (89.7\%). For petrous apex type PCMs, 16 cases (100\%) had total resection. For upper clivus type PCMs, total resection was achieved in 26 cases $(76.5 \%)$. For tentorium type PCMs, 15 cases achieved total resection, and 23 cases (60.5\%) subtotal resection. Cavernous type PCMs was characterized as the tight adhesion between the tumor and adjacent nerves and vessels in the cavernous sinus. Ten cases $(52.6 \%)$ were achieved subtotal resection and 9 cases (47.4\%) merely partial resection.

Another difficulty in the surgical management of PCMs is the intraoperative protection of cranial nerves $(25,26)$. A most frequent complication for any skull base approach is the ever-present risk of the injury to the CNs. According to the literature, the incidence of cranial nerve dysfunction after surgery is $20-100 \%(27-29)$. In this study, preoperative cranial nerve dysfunction mainly involved the III-IX cranial nerves. The incidence of new-onset and aggravated cranial nerve dysfunction were $13.1 \%(14 / 107)$ and $10.4 \%(15 / 144)$, respectively. Most neurological disorders were improved during the follow-up. For intraoperative neurological protection, the experience of the investigators was as follows: (1) The trigeminal nerve is located below the superior petrosal sinus, thus the cauterization of superior petrosal sinus should be given with more attention. The trochlear nerve is often located in the medial of the tumor, and the facial nerve and vestibular nerve are located on the lower lateral side of the tumor, the separation of them from the tumor should along the arachnoid membrane interface hence. (2) If the cranial nerves were tightly enclosed, such as III-VI nerves in cavernous type PCMs, the cranial nerve dysfunction is usually aggravated postoperatively. Therefore, the goal of surgery has 
been transferred from the total resection to maximum preservation, since preserving neurological functions is pivotal to improve postoperative quality of life. Thus, we recommend incomplete resection followed by adjuvant radiotherapy for this type PCMs (3). Serviceable hearing preservation is also very important. The hearing protection during tumor resection is mainly the protection of the auditory nerve. The same as for vestibular schwannomas, the translabyrinthine approach (labyrinthectomy) sacrifices hearing to achieve greater exposure and total resection, whereas the middle fossa approach (such as STA and KA) and retrosigmoid approach offer the possibility of hearing preservation (30). This highly influences the choice of surgical approaches: if PCM patients have practical hearing before surgery, the trans-middle cranial fossa approaches and retrosigmoid sinus approach can be used; if the patient does not have practical hearing before surgery, the translabyrinthine approach may be considered based on the tumor location. However, for PCMs, patients often suffer from cranial nerve dysfunction in $\mathrm{CN} \mathrm{V}$ and posterior group cranial nerves; the vestibulocochlear nerve complex often located caudally, making it a crucial maneuver to keep an intact arachnoid plane between the tumor and the surrounding structures. Under the protection of electrophysiological testing, the in-capsule tumor decompression should be implemented, and then the sharp separation between the residual envelope from the surrounding structures upon the arachnoid interface. The complete arachnoid interface must be ensured, so that the maximum tumor resection and hearing preservation can be achieved. In this study, most patients with preoperative hearing impairment had an improvement significantly at follow-up. Therefore, we claim that meticulous techniques and the knowledge of microsurgical anatomy shall lead to feasible hearing preservation with maximum tumor removal under contemporary circumstances.

With respect to the other postoperative complications, there were 14 cases suffering intracranial infection, 9 cases had cerebrospinal fluid leakage, and 3 cases with postoperative hematoma (1 case needed second operation). And there were two deaths because of pneumonia and multiple organ failure after postoperative hematoma. It is clear that modern cranial base techniques and resection skills can significantly reduce the complications. Despite transient neurological deterioration that may occurred during early postoperative periods after total resection. In this group, the incidence of new-onset and aggravated cranial nerve dysfunction were $13.1 \%$ (14/107) and $10.4 \%(15 / 144)$, respectively. Though the mean preoperative and postoperative KPS scores were 80 (range 60-100 points) and 78.6 (range $0-100$ points) respectively, this was not statistically significant ( $t=-0.102, P=0.922)$. Furthermore, at their most recent follow-up, 57 cases (53.3\%) were stable with no worsened KPS, 36 cases $(33.6 \%)$ had improved, only 14 patients $(13.1 \%)$ had aggravated KPS score. In addition, the progression or recurrence rate was statistically significant among TR, STR and PR groups $(\mathrm{P}<0.05)$ and there was no statistically significance between the $\mathrm{WHO}$ grade and degree of tumor resection $(\mathrm{P}<0.05)$. Thus, we suggest total resection appears to be advantageous for various skull base approaches on PCMs. This is consistent with Almefty et al. (1) who concluded that multiple skull base approaches to PCMs not only facilitate an improved chance of total resection, but also decrease the risk of morbidity. In our study, there was a statistically significance of the progression or recurrence rate when WHO grade I compared with WHO grade II and III, but it was insignificant between WHO grade II and III, which might be due to the invasive nature of grade II and III tumors, or simply the bias caused by the small amount of the two groups and the shortness of follow-up time.

\section{CONCLUSIONS}

In conclusion, resection of PCMs remains a challenge. The optimal surgical approach depends on the size, extension of the tumor and the anatomical relationship between the tumor and the cranial nerves. RSA and petrosal approaches were the most commonly used. With elaborate surgical plans and advanced microsurgical skills, most patients with PCMs can be rendered tumor resection with satisfactory extent and functional preservation, despite transient neurological deterioration during early postoperative periods.

\section{DATA AVAILABILITY STATEMENT}

The original contributions presented in the study are included in the article/supplementary material. Further inquiries can be directed to the corresponding authors.

\section{ETHICS STATEMENT}

The studies involving human participants were reviewed and approved by the Ethics Committee of the First People's Hospital of Yunnan Province. The patients/participants provided their written informed consent to participate in this study.

\section{AUTHOR CONTRIBUTIONS}

BG initiated the study and wrote the manuscript. YZ and JT collected the data. JO, BT, and XC analyzed the data. TL and SH coordinated the study and revised the article. All authors contributed to the article and approved the submitted version.

\section{FUNDING}

This work was supported by National Natural Science Foundation of China (Grant NO. 82001278), The Fund for Young Doctors with the First People's Hospital of Yunnan Province (Grant NO. KHBS-2020-014), Yunnan Fundamental Research Projects (Grant NO. 202101AU070106), and Joint Projects of Yunnan Provincial Science and Technology Department and Kunming Medical University for Applied Basic Research (Grant NO. 202101AY070001-252). 


\section{REFERENCES}

1. Almefty R, Dunn IF, Pravdenkova S, Abolfotoh M, Al-Mefty O. True Petroclival Meningiomas: Results of Surgical Management. J Neurosurg (2014) 120(1):40-51. doi: 10.3171/2013.8.JNS13535

2. Bhattacharya RN. Large-To-Giant Petroclival Meningiomas: The Additional Features in Management. Neurol India (2018) 66(5):1314-5. doi: 10.4103/ 0028-3886.241381

3. Kim JW, Jung HW, Kim YH, Park CK, Chung HT, Paek SH, et al. Petroclival Meningiomas: Long-Term Outcomes of Multimodal Treatments and Management Strategies Based on 30 Years of Experience at a Single Institution. J Neurosurg (2019) 132(6):1675-82. doi: 10.3171/2019.2.JNS182604

4. Shibao S, Borghei-Razavi H, Orii M, Yoshida K. Anterior Transpetrosal Approach Combined With Partial Posterior Petrosectomy for Petroclival Meningiomas With Posterior Extension. World Neurosurg (2015) 84(2):5749. doi: 10.1016/j.wneu.2015.03.055

5. Steiger HJ. Surgical Management of Medium and Large Petroclival Meningiomas: Benchmark and Limits. Acta Neurochir (Wien) (2016) 158 (3):407-8. doi: 10.1007/s00701-015-2686-Z

6. Lin $\mathrm{Y}, \mathrm{Gao}$ Q, Jin H, Wang N, Xu D, Wang F, et al. Analysis of Approaches in the Microsurgical Treatment of 102 Cases of Petroclival Meningioma in a Single Center. Front Neurol (2021) 12:627736. doi: 10.3389/fneur.2021.627736

7. Kawase T. Advantages and Disadvantages of Surgical Approaches to Petroclival Lesions. World Neurosurg (2011) 75(3-4):421. doi: 10.1016/j.wneu.2010.12.045

8. Kawase T, Shiobara R, Ohira T, Toya S. Developmental Patterns and Characteristic Symptoms of Petroclival Meningiomas. Neurol Med Chir (Tokyo) (1996) 36(1):1-6. doi: 10.2176/nmc.36.1

9. Ichimura S, Kawase T, Onozuka S, Yoshida K, Ohira T. Four Subtypes of Petroclival Meningiomas: Differences in Symptoms and Operative Findings Using the Anterior Transpetrosal Approach. Acta Neurochir (Wien) (2008) 150(7):637-45. doi: 10.1007/s00701-008-1586-x

10. Sekhar LN, Schramm VLJr, Jones NF. Subtemporal-Preauricular Infratemporal Fossa Approach to Large Lateral and Posterior Cranial Base Neoplasms. J Neurosurg (1987) 67(4):488-99. doi: 10.3171/jns.1987.67.4.0488

11. Natarajan SK, Sekhar LN, Schessel D, Morita A. Petroclival Meningiomas: Multimodality Treatment and Outcomes at Long-Term Follow-Up. Neurosurgery (2007) 60(6):965-79; discussion 979-81. doi: 10.1227/ 01.NEU.0000255472.52882.D6

12. Li D, Hao SY, Wang L, Tang J, Xiao XR, Jia GJ, et al. Recurrent Petroclival Meningiomas: Clinical Characteristics, Management, and Outcomes. Neurosurg Rev (2015) 38(1):71-86; discussion 86-7. doi: 10.1007/s10143014-0575-1

13. Li D, Hao SY, Wang L, Tang J, Xiao XR, Zhou H, et al. Surgical Management and Outcomes of Petroclival Meningiomas: A Single-Center Case Series of 259 Patients. Acta Neurochir (Wien) (2013) 155(8):1367-83. doi: 10.1007/s00701013-1795-9

14. Li D, Tang J, Ren C, Wu Z, Zhang LW, Zhang JT. Surgical Management of Medium and Large Petroclival Meningiomas: A Single Institution's Experience of 199 Cases With Long-Term Follow-Up. Acta Neurochir (Wien) (2016) 158 (3):409-25; discussion 425. doi: 10.1007/s00701-015-2671-6

15. Li P, Yang Z, Wang Z, Zhou Q, Li S, Wang X, et al. Clinical Features of Clear Cell Meningioma: A Retrospective Study of 36 Cases Among 10,529 Patients in a Single Institution. Acta Neurochir (Wien) (2016) 158(1):67-76. doi: 10.1007/s00701-015-2635-x

16. Bambakidis NC, Kakarla UK, Kim LJ, Nakaji P, Porter RW, Daspit CP, et al. Evolution of Surgical Approaches in the Treatment of Petroclival Meningiomas: A Retrospective Review. Neurosurgery (2008) 62(6 Suppl 3):1182-91. doi: 10.1227/ 01.NEU.0000333784.04435.65

17. Borghei-Razavi H, Tomio R, Fereshtehnejad SM, Shibao S, Schick U, Toda M, et al. Anterior Petrosal Approach: The Safety of Kawase Triangle as an Anatomical Landmark for Anterior Petrosectomy in Petroclival Meningiomas. Clin Neurol Neurosurg (2015) 139:282-7. doi: 10.1016/j.clineuro.2015.10.032
18. Altieri R, Sameshima T, Pacca P, Crobeddu E, Garbossa D, Ducati A, et al. Detailed Anatomy Knowledge: First Step to Approach Petroclival Meningiomas Through the Petrous Apex. Anat Lab Experience Surg series Neurosurg Rev (2017) 40(2):231-9. doi: 10.1007/s10143-016-0754-3

19. Calbucci F. Treatment Strategy for Sphenopetroclival Meningiomas. World Neurosurg (2011) 75(3-4):419-20. doi: 10.1016/j.wneu.2010.12.033

20. Morisako H, Goto T, Ohata K. Petroclival Meningiomas Resected via a Combined Transpetrosal Approach: Surgical Outcomes in 60 Cases and a New Scoring System for Clinical Evaluation. J Neurosurg (2015) 122(2):37380. doi: 10.3171/2014.8.JNS132406

21. Wayhs SY, Lepski GA, Frighetto L, Isolan GR. Petroclival Meningiomas: Remaining Controversies in Light of Minimally Invasive Approaches. Clin Neurol Neurosurg (2017) 152:68-75. doi: 10.1016/j.clineuro.2016.11.021

22. Isolan GR, Wayhs SY, Lepski GA, Dini LI, Lavinsky J. Petroclival Meningiomas: Factors Determining the Choice of Approach. J Neurol Surg B Skull Base (2018) 79:367-78. doi: 10.1055/s-0037-1608654

23. Almefty KK, Al-Mefty O. Petrosal Approach With Preservation of the Superior Petrosal Sinus (the Graceful Petrosal) for Resection of Giant Trigeminal Schwannoma: 2-Dimensional Operative Video. Oper Neurosurg (Hagerstown) (2021) 20:E342-342E343. doi: 10.1093/ons/opaa427

24. Hafez A, Nader R, Al-Mefty O. Preservation of the Superior Petrosal Sinus During the Petrosal Approach. J Neurosurg (2011) 114:1294-8. doi: 10.3171/ 2010.6.JNS091461

25. Maurer AJ, Safavi-Abbasi S, Cheema AA, Glenn CA, Sughrue ME. Management of Petroclival Meningiomas: A Review of the Development of Current Therapy. J Neurol Surg B Skull Base (2014) 75(5):358-67. doi: 10.1055/s-0034-1373657

26. Beniwal M, Bhat DI, Rao N, Bhagavatula ID, Somanna S. Surgical Management of Petroclival Meningiomas: Factors Affecting Early Post-Operative Outcome. Br J Neurosurg (2015) 29(4):559-64. doi: 10.3109/02688697.2015.1019422

27. Couldwell WT, Fukushima T, Giannotta SL, Weiss MH. Petroclival Meningiomas: Surgical Experience in 109 Cases. J Neurosurg (1996) 84 (1):20-8. doi: 10.3171/jns.1996.84.1.0020

28. Little KM, Friedman AH, Sampson JH, Wanibuchi M, Fukushima T. Surgical Management of Petroclival Meningiomas: Defining Resection Goals Based on Risk of Neurological Morbidity and Tumor Recurrence Rates in 137 Patients. Neurosurgery (2005) 56(3):546-59. doi: 10.1227/ 01.NEU.0000153906.12640.62

29. Seifert V. Clinical Management of Petroclival Meningiomas and the Eternal Quest for Preservation of Quality of Life: Personal Experiences Over a Period of 20 Years. Acta Neurochir (Wien) (2010) 152(7):1099-116. doi: 10.1007/s00701-010-0633-6

30. Raheja A, Bowers CA, MacDonald JD, Shelton C, Gurgel RK, Brimley C, et al. Middle Fossa Approach for Vestibular Schwannoma: Good Hearing and Facial Nerve Outcomes With Low Morbidity. World Neurosurg (2016) 92:3746. doi: 10.1016/j.wneu.2016.04.085

Conflict of Interest: The authors declare that the research was conducted in the absence of any commercial or financial relationships that could be construed as a potential conflict of interest.

Publisher's Note: All claims expressed in this article are solely those of the authors and do not necessarily represent those of their affiliated organizations, or those of the publisher, the editors and the reviewers. Any product that may be evaluated in this article, or claim that may be made by its manufacturer, is not guaranteed or endorsed by the publisher.

Copyright () 2021 Gao, Zhang, Tan, Ouyang, Tai, Cao, Li and Hu. This is an openaccess article distributed under the terms of the Creative Commons Attribution License (CC BY). The use, distribution or reproduction in other forums is permitted, provided the original author(s) and the copyright owner(s) are credited and that the original publication in this journal is cited, in accordance with accepted academic practice. No use, distribution or reproduction is permitted which does not comply with these terms. 